(C) Copyright 2012 American Meteorological Society (AMS). Permission to use figures, tables, and brief excerpts from this work in scientific and educational works is hereby granted provided that the source is acknowledged. Any use of material in this work that is determined to be "fair use" under Section 107 of the U.S. Copyright Act or that satisfies the conditions specified in Section 108 of the U.S. Copyright Act (17 USC §108, as revised by P.L. 94-553) does not require the AMS's permission. Republication, systematic reproduction, posting in electronic form, such as on a web site or in a searchable database, or other uses of this material, except as exempted by the above statement, requires written permission or a license from the AMS. Additional details are provided in the AMS Copyright Policy, available on the AMS Web site located at (http://www.ametsoc.org/) or from the AMS at 617-227-2425 or copyright@ametsoc.org. 


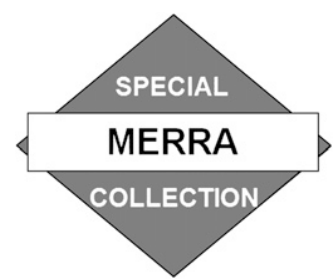

\title{
Object-Based Evaluation of MERRA Cloud Physical Properties and Radiative Fluxes during the 1998 El Niño-La Niña Transition
}

\author{
DEREK J. POSSELT \\ University of Michigan, Ann Arbor, Michigan \\ ANDREW R. JONGEWARD \\ University of Maryland, College Park, College Park, Maryland \\ Chuan-Yuan Hsu and Gerald L. Potter \\ University of Michigan, Ann Arbor, Michigan
}

(Manuscript received 8 December 2011, in final form 7 May 2012)

\begin{abstract}
The Modern-Era Retrospective Analysis for Research and Application (MERRA) is a reanalysis designed to produce an improved representation of the Earth's hydrologic cycle. This study examines the representation of deep convective clouds in MERRA, comparing analyzed liquid and ice clouds with deep convective cloud objects observed by instruments on the Tropical Rainfall Measuring Mission satellite. Results show that MERRA contains deep convective cloud in $98.1 \%$ of the observed cases. MERRA-derived probability density functions (PDFs) of cloud properties have a similar form as the observed PDFs and exhibit a similar trend with changes in object size. Total water path, optical depth, and outgoing shortwave radiation (OSR) in MERRA are found to match the cloud object observations quite well; however, there appears to be a bias toward higher-than-observed cloud tops in the MERRA. The reanalysis fits the observations most closely for the largest class of convective systems, with performance generally decreasing with a transition to smaller convective systems. Comparisons of simulated total water path, optical depth, and OSR are found to be highly sensitive to the assumed subgrid distribution of condensate and indicate the need for caution when interpreting model-data comparisons that require disaggregation of grid-scale cloud to satellite pixel scales.
\end{abstract}

\section{Introduction}

In recent decades, reanalysis has emerged as an effective way to combine numerical model output with information from a diverse suite of atmospheric state measurements such that the proper relationships between dynamics, thermodynamics, radiation, and surface fluxes are preserved (Kalnay et al. 1996; Uppala et al. 2005; Onogi et al. 2005; Rienecker et al. 2011). In large part because these datasets provide a long-term dynamically consistent and multivariate representation of a host of Earth system variables, reanalyses have been

Corresponding author address: Derek J. Posselt, Department of Atmospheric, Oceanic and Space Sciences, University of Michigan, 2455 Hayward Street, Ann Arbor, MI 48109-2143.

E-mail: dposselt@umich.edu successfully used in a wide range of weather and climate research activities. The general utility of a reanalysis dataset depends on the fidelity of its representation of the state of the atmosphere, which in turn depends on the data assimilation algorithm, data assimilated, and realism of the numerical model's physical parameterizations. While most reanalysis efforts produce robust estimates of temperature and horizontal wind components, water vapor, clouds, and precipitation have proven more difficult to analyze accurately. The importance of characterizing cloud feedbacks in the climate system (Stephens 2005) and increasing understanding of flooddrought cycles and extreme precipitation events has led to an increased focus on the realism of the hydrologic cycle in reanalyses (Rienecker et al. 2011).

The Modern-Era Retrospective Analysis for Research and Application (MERRA) is a reanalysis specifically 
designed to improve on estimates of the hydrologic cycle via assimilation of moisture tendencies derived from observations of precipitation (Rienecker et al. 2008, 2011; Robertson et al. 2011). MERRA is unique among modern reanalyses, in that it includes many atypical model output fields (e.g., tendencies from model physical parameterizations and analysis increments from the data assimilation scheme) and reports output at high temporal and spatial resolution. An overview of MERRA is provided by Rienecker et al. (2011), and an evaluation of the global water and energy budget is presented in Bosilovich et al. (2011). Examination of the long-term mean state indicates that, while MERRA tends to overestimate tropical precipitation and underestimate precipitation in the midlatitudes, the biases in MERRA are smaller than those in other modern reanalysis datasets. Overall, MERRA provides significant improvements in the fidelity of the hydrologic cycle, as compared with previous reanalyses (Rienecker et al. 2011).

While production of realistic long-term and largescale cloud physical and radiative properties is a firstorder requirement for a reanalysis dataset, it is also important to evaluate the detailed properties of analyzed clouds. In the Earth's climate system, cloud scales are coupled to synoptic and planetary scales, and the bulk of the climate-relevant cloud feedbacks depend on changes in the nature of the cloud response to the evolving large-scale circulation and environment. One of the most robustly coupled features in the climate system is the persistent deep convection present in the ascending branch of the Hadley-Walker circulation. The strength and location of the Walker circulation, in particular, is strongly modulated by the cyclic changes in the El Niño-Southern Oscillation (ENSO). One of the strongest recorded El Niño events occurred in 1997/98 (Bell et al. 1999) during the midst of the MERRA period, and was associated with a shift in the Walker circulation from the western to central Pacific. Almost as remarkable as the strength of the event was the rapidity of its transition to a La Niña state in the middle of 1998. Cloud properties and radiative fluxes during the 1998 El NiñoLa Niña transition were observed by the Tropical Rainfall Measuring Mission (TRMM) satellite and the instruments on board have been used to identify sets of contiguous cloud features or "objects" in the data (Xu et al. 2005, 2007, 2008). A subset of these objects is associated with the presence of deep convection, and it has already been used to evaluate cloud structures in the European Centre for Medium-Range Weather Forecasts (ECMWF) operational analysis and the 40-yr ECMWF Re-Analysis (ERA-40; Xu 2009).

The benefit of an object-based analysis is the ability to study coherent structures. The wealth of information contained in the probability density function (PDF) of cloud properties provides the opportunity to examine how the details of deep convection change, and it lends itself naturally to a process-based statistical examination of both the properties of the system and the evaluation of large-scale models. In particular, collection of the sample of objects into a database of deep convective statistics makes it more straightforward to use in the analysis of general circulation model (GCM) output, for which it is not as important that each object be reproduced in its observed location but instead that the distribution of cloud and radiative properties be correct. Model evaluation that targets a specific process or set of processes has the potential to more effectively identify the source of persistent model biases. A more robust identification of the source of biases in GCM-simulated cloud-radiative feedbacks is a primary objective for the cloud objects database (Xu et al. 2005).

In this paper, we conduct a similar comparison as $\mathrm{Xu}$ (2009) for the purpose of evaluating the cloud properties and radiative fluxes in the MERRA data. We examine the ability of MERRA to successfully reproduce the properties of deep convective clouds in the tropical Pacific Ocean basin. We compare the reanalysis with TRMM-retrieved variables by transforming MERRA output into the same grid as the Clouds and the Earth's Radiant Energy System (CERES) data and then proceed to examine the effect of changes to assumptions inherent in the transformation from model space to observation space. The remainder of this paper is structured as follows. Section 2 contains a description of the CERES cloud object data as well as an overview of the deep convective object locations and bulk properties for the 1998 El Niño-La Niña transition. Section 3 details the method used to transform model output into observation space and explores the potential pitfalls in such a transformation. Results of the comparison between MERRA and cloud object data are presented in section 4, and the effect of changes to the assumptions used in the transformation of model to observation space is explored in section 5. A brief summary and conclusions can be found in section 6 .

\section{CERES deep convective cloud object data}

Xu et al. $(2005,2007,2008)$ used data from multiple instruments on the TRMM satellite (Kummerow et al. 1998) to identify contiguous cloudy regions over the tropical Pacific Ocean basin, and to classify cloud systems into four different categories. The area chosen for the examination of deep convective clouds in particular extended from $25^{\circ} \mathrm{S}$ to $25^{\circ} \mathrm{N}$ latitude and from $130^{\circ} \mathrm{E}$ to $80^{\circ} \mathrm{W}$ longitude. Broadband radiances from 

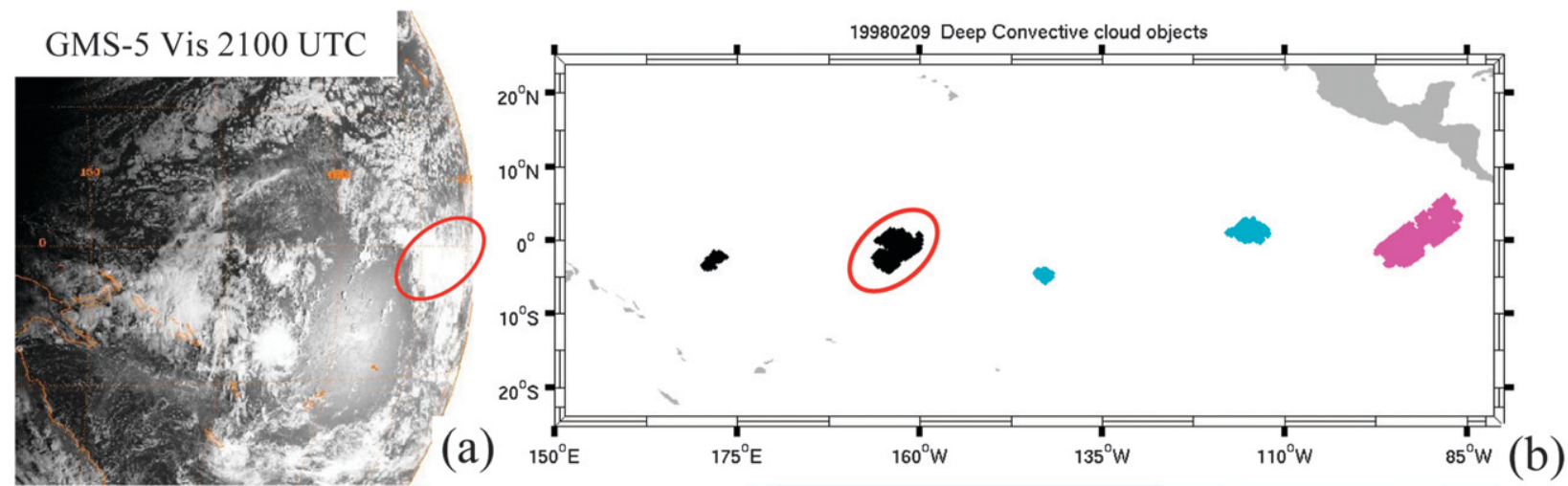

CERES Footprints

PDF: Optical Depth

PDF: Outgoing LW

PDF: Outgoing SW
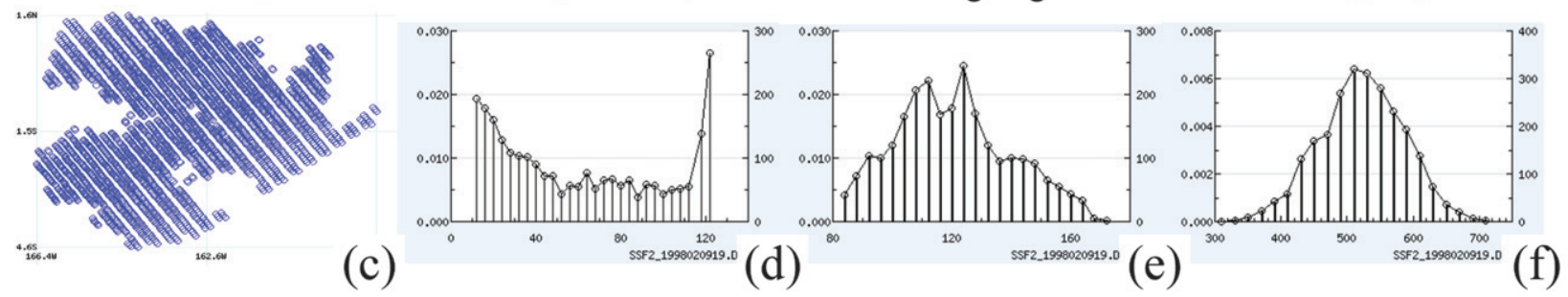

FIG. 1. Sample deep convective cloud object observed at 1900 UTC 9 Feb 1998 . Shown are (a) the GMS-5 visible imagery, (b) cloud object mask, (c) CERES footprints, and PDFs of (d) optical depth, (e) OLR, and (f) OSR.

the version 2B CERES Single Satellite Footprint (SSF) data (Wielicki et al. 1996) were used to characterize topof-atmosphere (TOA) outgoing broadband longwave and shortwave radiative fluxes on CERES $10-\mathrm{km}$ footprints. Cloud macro- and microphysical information were retrieved from the Visible and Infrared Scanner (VIRS; Minnis et al. 2011a,b) and energy was weighted over the larger CERES footprints according to the CERES instrument energy point spread function (Minnis et al. 1997). CERES on TRMM was only operational during January-August 1998 and March 2000; however, these periods correspond to the rapid 1998 El Niño-La Niña transition during January-August 1998 and to La Niña conditions during March 2000. A detailed description of the TRMM instrument packages can be found in Kummerow et al. (1998) and the CERES SSF data are documented online (at http://ceres.larc.nasa.gov/products. php? product $=$ SSF-Level2).

Cloud objects are defined by Xu et al. (2005, 2007, 2008) to be coherent regions of contiguous cloud with properties that conform to a set of cloud system type criteria. Thresholds on cloud-top height, optical depth, and cloud fraction (determined from higher spatial resolution VIRS data within each footprint) are used to identify cloud objects, and each CERES field of view is classified according to type. A search algorithm is then applied that identifies adjacent pixels with similar properties, and the cloud object is allowed to grow until no further pixels with the candidate criteria are found. Cloud macro- and microphysical properties within each CERES footprint are obtained from VIRS. In this study, we focus on deep convective cloud objects, which are defined in the CERES SSF data to have cloud-top height greater than $10 \mathrm{~km}$ and cloud optical depth greater than $10 \mathrm{~km}$. A sample deep convective object observed on 9 February 1998 (Fig. 1) demonstrates the information contained in each object. It is clear from the Japanese Geostationary Meteorological Satellite-5 (GMS-5) imagery (Fig. 1a) that objects are coherent entities, and (from the object mask in Figs. 1b and 1c) that the object identification code effectively isolates contiguous pixels with deep convective characteristics. The fact that the maximum value of the outgoing longwave radiation (OLR) in the object (Fig. 1e) is on the order of $170 \mathrm{~W} \mathrm{~m}^{2}$ makes it clear that the tops of observed clouds extend into the upper troposphere. Objects are characterized by significant internal variability, reflecting the presence of both convective cores and stratiform anvil (Figs. 1d-f).

Plots of the location of each deep convective cloud object for each month during January-August 1998 (Fig. 2) demonstrate the correspondence between the location of the deep convective cloud objects and the location of the intertropical convergence zone (ITCZ) and ascending branch of the Walker circulation. Consistent with results presented in Xu et al. (2007), there is little apparent change in the distribution of object sizes 

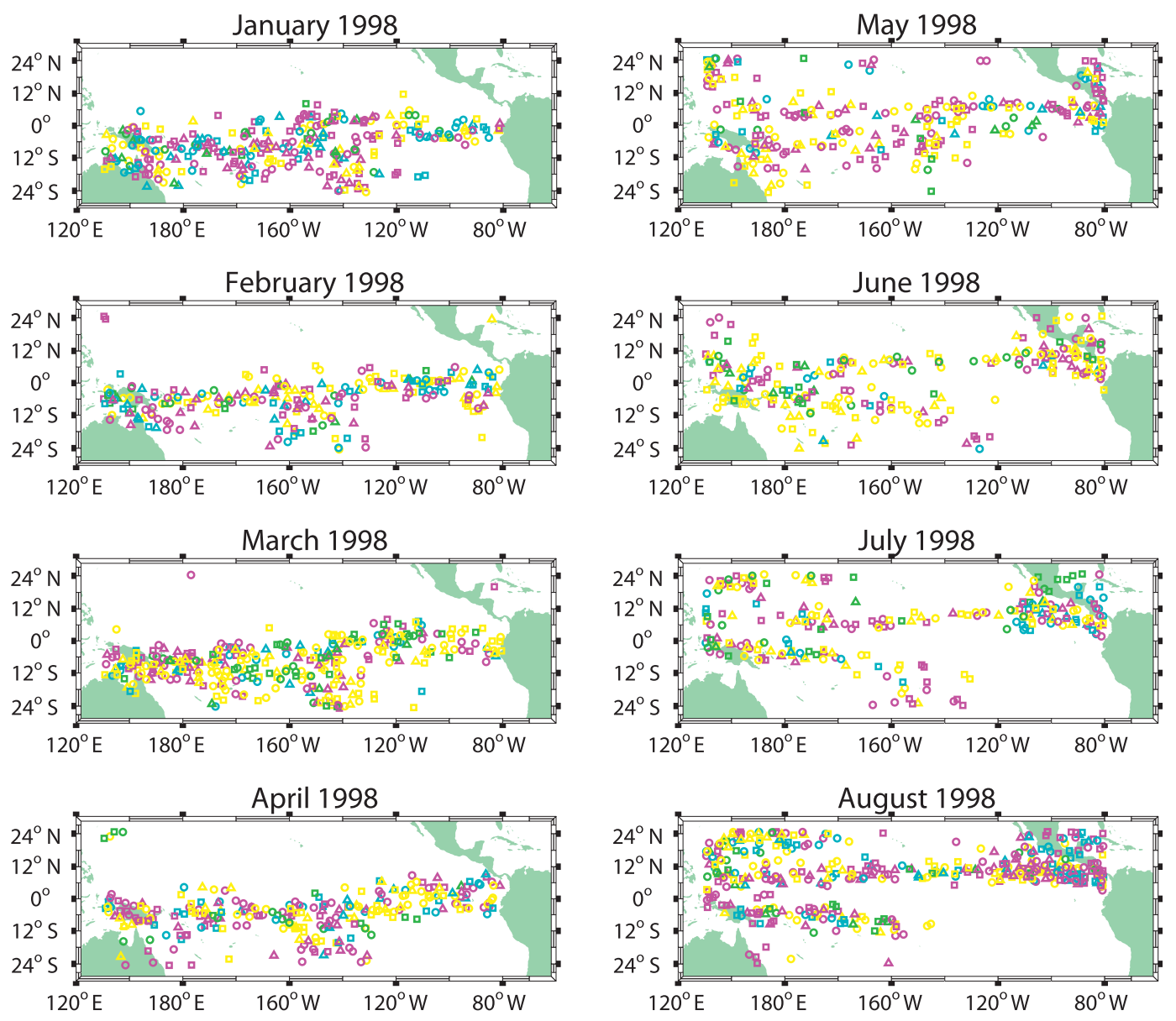

FIG. 2. Object locations and sizes for each month in the January-August 1998 period. Symbols depict the object size with squares representing objects between 100 and $150 \mathrm{~km}$ in diameter, circles objects between 150 and $300 \mathrm{~km}$ in diameter, and triangles objects $>300 \mathrm{~km}$ in diameter. Colors depict the local standard time of occurrence of each object with green representing 0600-0900 local standard time (LST), yellow 0900-1200 LST, magenta 1200-1500 LST, and blue-green 1500-1800 LST.

with the transition from El Niño to La Niña. The effect of El Niño on the location of deep convection can be seen in an analysis of the fraction of deep convective objects located over the western, central, or eastern Pacific Ocean (Fig. 3). There is a nearly monotonic decrease with time in the fraction of the total number of objects located in the central Pacific. At the same time, as the fraction of objects located in the western Pacific increases, so does the number in the eastern Pacific.

In separating clouds into coherent structures according to type, the cloud object database offers the opportunity to perform a statistical and process-based evaluation of analyzed clouds in the MERRA dataset. Limitations of the CERES cloud object dataset include the use of VIRS to constrain the cloud properties, which restricts the objects to times when there is sufficient solar reflection (e.g., a range of solar zenith angle between $0^{\circ}$ and $60^{\circ}$ ). In addition, the information content of VIRS for cloud liquid and ice path is relatively low compared with more modern sensors [e.g., the Moderate Resolution Imaging Spectroradiometer (MODIS)]. Finally, the need to first disaggregate model grid cells into single-scanner footprint pixels and then to extract subcolumns with deep convective properties adds another source of uncertainty to the comparison. We explore the implications of this later in section 5 .

\section{Comparison between MERRA and cloud objects: The method}

For each object in the CERES cloud object database, we extract the corresponding MERRA grid boxes at the nearest 3-hourly output time. To account for the possibility of movement of the analyzed cloud system, we expand the comparison region to include a one-grid-box window around each object. MERRA output directly 


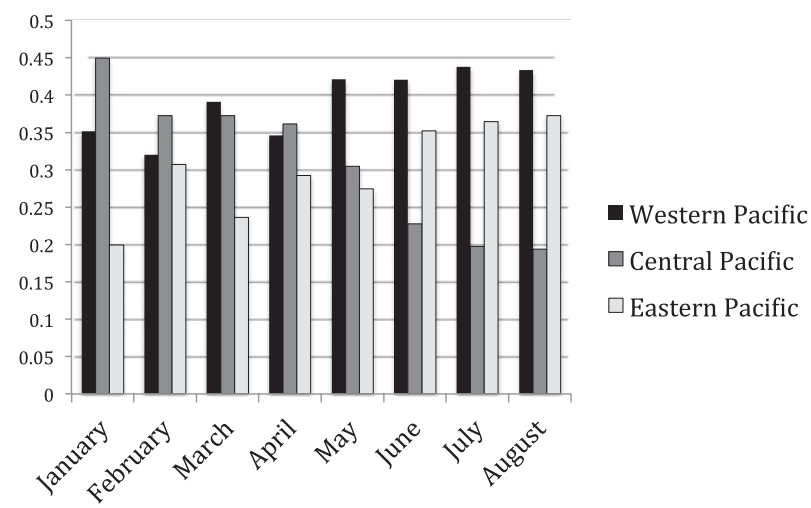

FIG. 3. Fraction of deep convective cloud objects observed by CERES in the western, eastern, and central Pacific Ocean over the period January-August 1998.

used in our comparison includes the convective and largescale liquid and ice mass mixing ratio, convective and large-scale cloud fraction, and downward fluxes of liquid-and-ice-phase precipitation through each layer boundary. In addition, surface pressure and skin temperature, as well as three-dimensional temperature, geopotential height, and water vapor, are used in the calculation of subgrid-scale optical depth and radiative fluxes.

CERES SSF data are on $10 \mathrm{~km} \times 10 \mathrm{~km}$ pixels, while MERRA is run nominally on a $0.5^{\circ} \times 0.625^{\circ}$ grid. However, information needed to effectively compare the reanalysis with observations (e.g., three-dimensional cloud fraction) is only available in complete form on a $1.0^{\circ} \times$ $1.25^{\circ}$ grid. As such, an effective comparison between MERRA and pixel-level cloud object data requires disaggregation of reanalysis fields into subcolumns. A similar exercise is performed when comparing model output to First International Satellite Cloud Climatology Project (ISCCP) data (Bodas-Salcedo et al. 2008) and has been described for evaluation of ECMWF model fields using cloud object data in Xu (2009). To disaggregate MERRA fields onto 10-km CERES pixels, we employ the subcolumn generator used in version 1.3 of the Cloud Feedback Model Intercomparison Project (CFMIP) Observation Simulator Package (COSP; Bodas-Salcedo et al. 2008). Clouds in adjacent layers are assumed to be maximally overlapped, while contiguous layers of cloud in the vertical are assumed to be randomly overlapped. Both convective and large-scale cloud fraction are input to the subcolumn generator, and convective and stratiform clouds are treated separately. Output from the COSP subcolumn generator is a flag for each $10 \mathrm{~km} \times$ $10 \mathrm{~km}$ MERRA subgrid column, indicating whether a particular level in each column contains convective or large-scale cloud or clear air. Condensate produced by the convective cloud scheme is assigned to convective subcolumns, while grid-scale cloud mass is spread among stratiform subcolumns. To compute the subgrid distribution of water content and the associated radiative fluxes, the distribution of cloud mass across subcolumns is required. Consistent with the assumptions made in the COSP, liquid and ice cloud mass is assumed to be evenly distributed among all cloudy subcolumns. This assumption is valid if the cloud can be assumed to be horizontally uniform across the grid box. Grid boxes containing a mix of deep convective cores and stratiform anvil may have a highly nonuniform distribution of cloud mass, and we explore the sensitivity of the output to the assumed subgrid cloud mass distribution later in section 5 .

Calculation of broadband longwave and shortwave radiative fluxes is performed using the $\mathrm{Fu}$-Liou radiative transfer model (Fu and Liou 1992), which requires as input profiles of liquid and ice water content, temperature, water vapor mixing ratio, and ozone. It is assumed that temperature, pressure, ozone concentration, and vapor mixing ratio are homogeneous within each MERRA grid box. Surface infrared emissivity and shortwave albedo are obtained from the MERRA dataset, as well as surface skin temperature and pressure. Cloud liquid effective radius was set constant at $10 \mu \mathrm{m}$, and ice effective diameter $\left(D_{i}\right)$ was computed as a function of the layer ice water path (IWP) following the method described by Heymsfield et al. (2003). We have chosen to use Heymsfield et al.'s (2003) general fit because of its applicability to a wider range of ice water path values. Suspended rain and snow were estimated from the fluxes of liquid and ice precipitation through each model layer boundary, and the particle size distribution for each was assumed to follow a truncated constant-slope gamma function (Manton and Cotton 1977). Rain minimum, characteristic, and maximum radius were 60,162 , and $1800 \mu \mathrm{m}$, respectively, and snow minimum, characteristic, and maximum radii were 60,500 , and $5000 \mu \mathrm{m}$, respectively.

Once the set of MERRA grid boxes that surround each cloud object has been processed through the subcolumn generator and radiative transfer model, the subcolumn cloud-top height is defined to be the level at which the vertically integrated (from top down) cloud optical depth exceeds 1 . This is consistent with the definition of the cloud-top height used in the cloud object database. As in the CERES deep convective cloud objects, MERRA subcolumns with total optical depth less than 10 and with cloud-top height lower than $10 \mathrm{~km}$ are excluded from the comparison. After screening, it was found that there were 55 out of 2955 (1.9\%) objects for which MERRA did not contain any deep convective subcolumns. Following Xu (2009, see his Table 2), we 
TABLE 1. Rates of underestimated and overestimated deep convective MERRA subcolumns for small, medium, and large categories of cloud objects during January-August 1998. See text for details.

\begin{tabular}{lllllr}
\hline \hline \multirow{2}{*}{ Threshold } & \multicolumn{2}{c}{ Underestimate $(\%)$} & & \multicolumn{2}{c}{ Overestimate $(\%)$} \\
\cline { 2 - 3 } \cline { 5 - 6 } & $<10$ & $<20$ & & $>150$ & $>200$ \\
\hline Small & 2.91 & 3.99 & 28.22 & 25.79 \\
Medium & 2.34 & 3.52 & 25.82 & 22.06 \\
Large & 0.95 & 1.52 & 11.17 & 7.34 \\
\hline
\end{tabular}

computed for each cloud object the ratio of the number of detected deep convective subcolumns in the MERRA grid boxes to the number of deep convective footprints in the CERES SSF data. Table 1 lists the percent of small-, medium-, and large-sized objects for which the MERRA made a significant underestimate [the number of deep convective subcolumns found in the MERRA grid cells encompassing the object were fewer than $10 \%$ (20\%) of the number of deep convective CERES footprints] or overestimate [the number of MERRA deep convective subcolumns was greater than $150 \%(200 \%)$ of the number of CERES footprints classified as deep convective]. MERRA produced a significant underestimate in a very small fraction of cases, and this number decreased with increasing object size to less than $1 \%$ for the largest objects. Perhaps partially because of the inclusion of a window region around each deep convective cloud
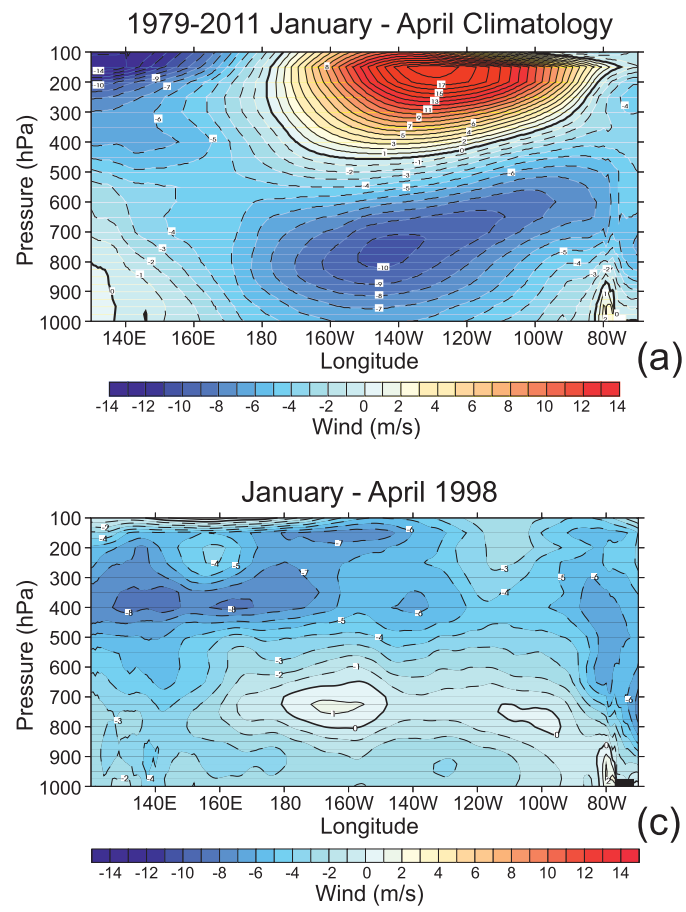

object, the number of cases for which MERRA produced an overestimate of the number of deep convective subcolumns was much larger; however, this number also decreased significantly with increasing object size. We tested the sensitivity of the results to changes in the screening procedure by varying the criteria for cloud detection, allowing a range of $9-11 \mathrm{~km}$ on the cloud-top height and 9-11 km on optical depth. There was negligible sensitivity in the results.

\section{Evaluation of MERRA for the 1998 El Niño-La Niña transition period}

\section{a. The large-scale response to changes in the Walker circulation}

Before entering into a detailed comparison between MERRA-simulated and TRMM-observed deep convective objects, we examine the large-scale characteristics of MERRA during the El Niño-La Niña transition and perform a comparison with the gridded monthlymean CERES radiative fluxes (data obtained from N. Loeb 2009, personal communication). The zonal velocity meridionally averaged over latitudes between $5^{\circ} \mathrm{N}$ and $5^{\circ} \mathrm{S}$ can be used to examine the approximate location and strength of the Walker circulation. A plot of this variable averaged over January-April (JFMA, Fig. 4a) and May-August (MJJA, Fig. 4b) for the 32-yr
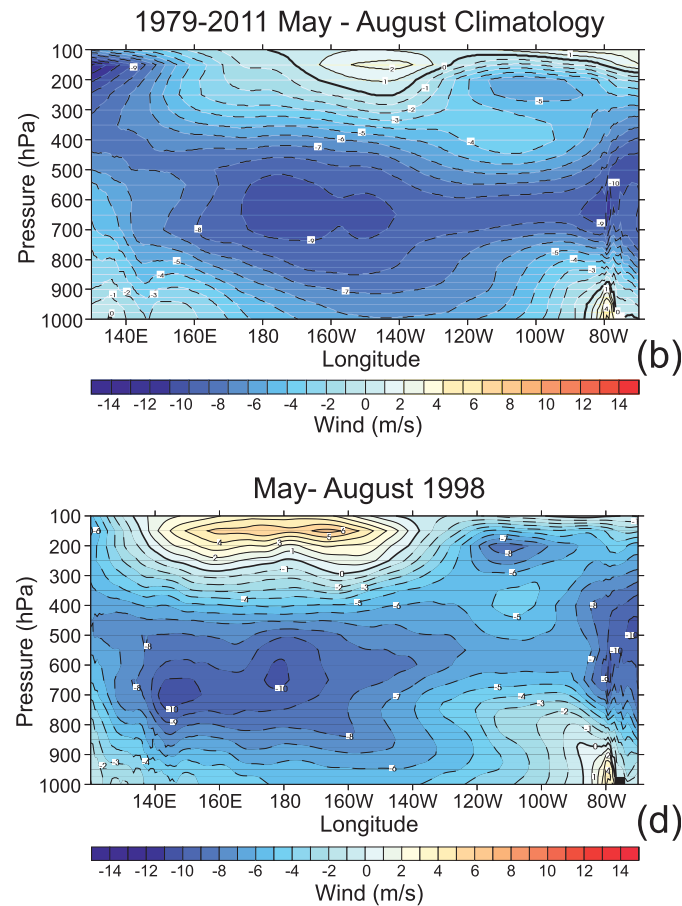

FIG. 4. Zonal velocity $\left(\mathrm{m} \mathrm{s}^{-1}\right)$ averaged over the $5^{\circ} \mathrm{N}-5^{\circ} \mathrm{S}$ latitude band for (a) every January-May in the 1979-2011 period, (b) every May-August in the 1979-2011 period, (c) January-April 1998, and (d) May-August 1998. 

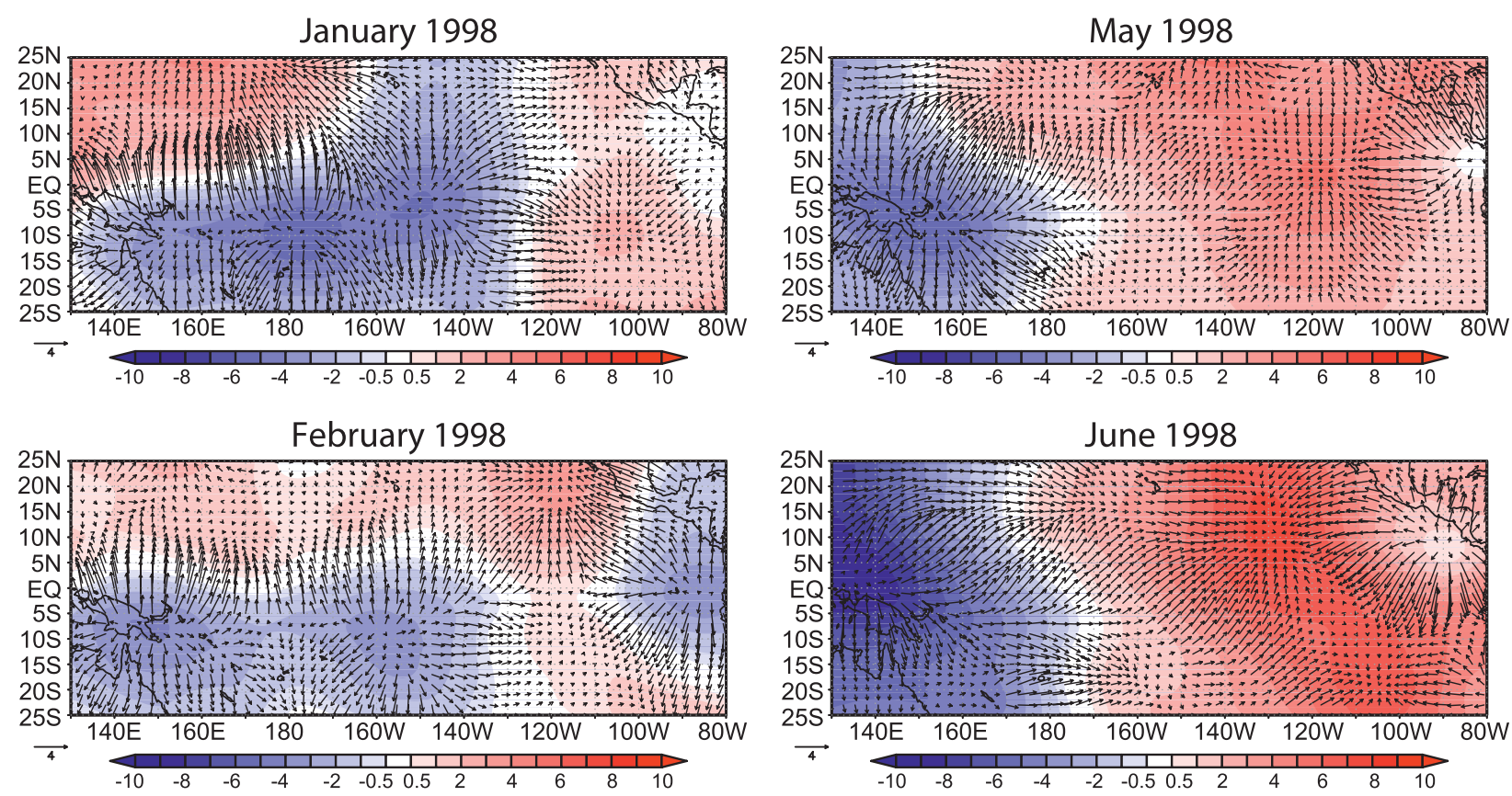

March 1998
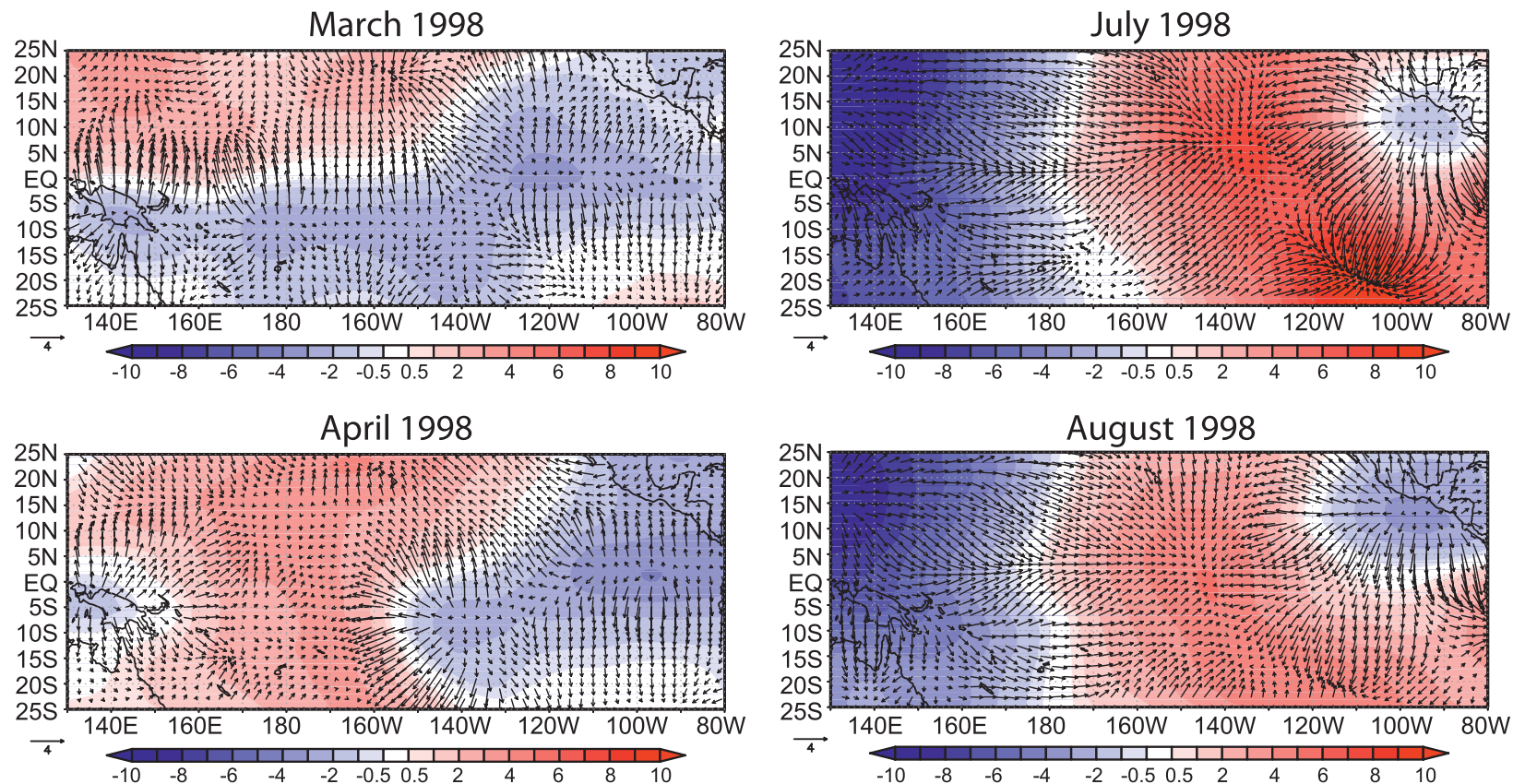

FIG. 5. MERRA-analyzed monthly-mean 250-hPa velocity potential and divergent winds $\left(\mathrm{m}^{2} \mathrm{~s}^{-1}\right.$ ) for (a) January, (b) February, (c) March, (d) April, (e) May, (f) June, (g) July, and (h) August 1998.

period between January 1979 and January 2011 clearly shows the Walker circulation signature, with convergence at low levels and divergence in the upper troposphere around $170^{\circ} \mathrm{E}$ longitude for JFMA and $150^{\circ} \mathrm{E}$ longitude for MJJA. Examination of the mean zonal wind speed from January to April 1998 (Fig. 4a) shows a near-complete collapse of the zonal overturning circulation. The same plot valid May-August 1998 (Fig. 4d) shows a rapid recovery, though the maximum in the lower-tropospheric easterlies is shifted approximately $30^{\circ}$ farther west than in the 32-yr mean, and the uppertropospheric westerlies are stronger and also shifted to the west.

The upper-tropospheric velocity potential (Fig. 5) and 500-hPa omega (Fig. 6) are consistent with the mean zonal overturning, showing a shift in the location of deep 


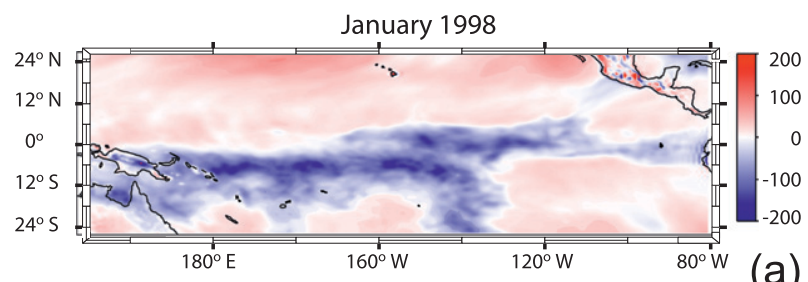

(a)

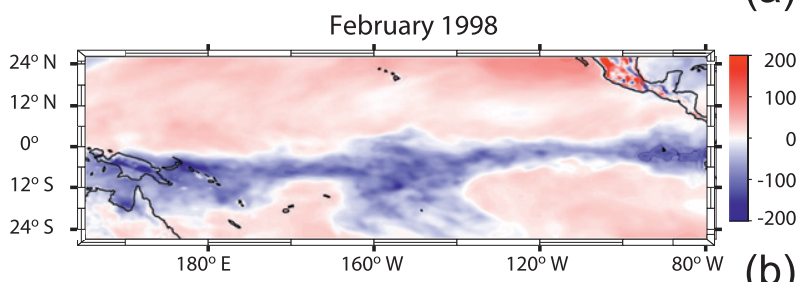

(b)
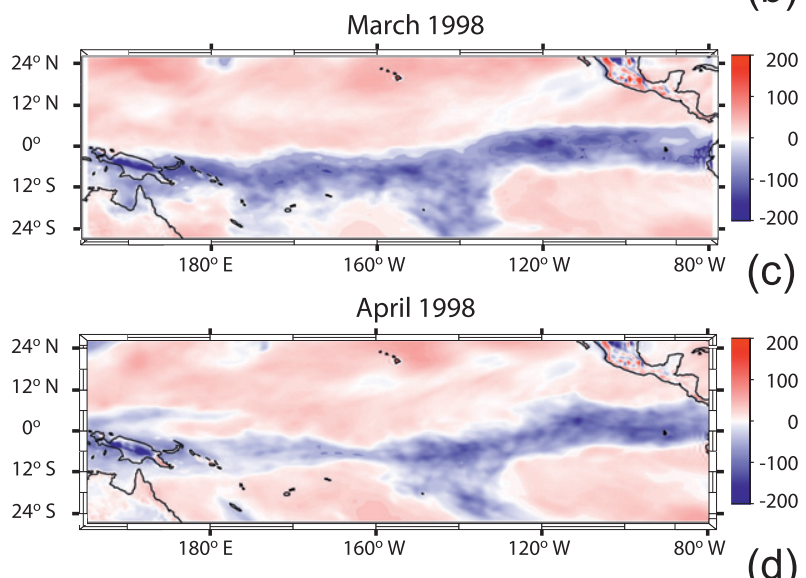

(d)
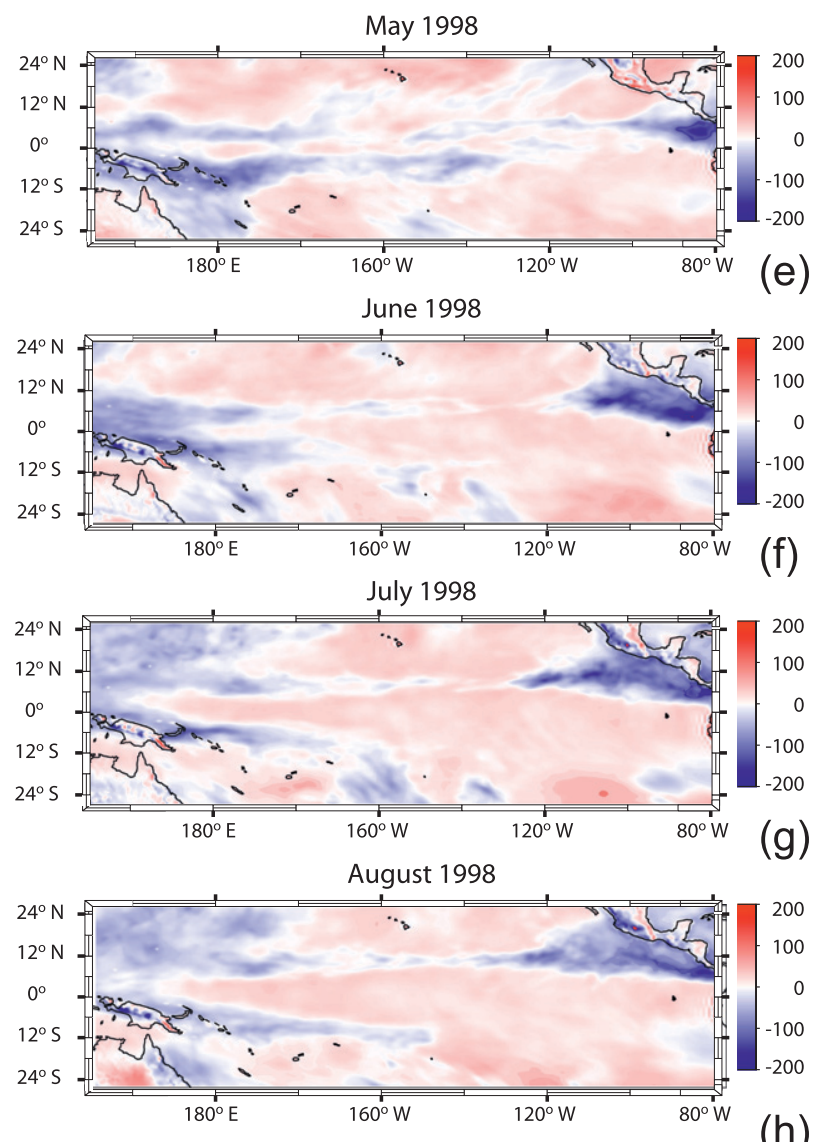

FIG. 6. As in Fig. 5, but for $500-\mathrm{hPa}$ omega $\left(\mathrm{hPa} \mathrm{day}^{-1}\right)$.

convection (and the rising branch of the Walker circulation) to the central Pacific with time. Note that the locations of deep convective cloud objects (Fig. 2) match closely with regions of upper-tropospheric divergence and midtropospheric rising motion in MERRA. The rapid shift in the location of deep convection from the central Pacific in April to the western Pacific in May is notable, and the concentration of deep convection in the central Pacific during January-April is consistent with the recent characterization of the $1998 \mathrm{El}$ Niño as a "central Pacific" event (Yeh et al. 2009). The shift in the Walker circulation can also be seen in the OLR averaged over JFMA and MJJA (Figs. 7a-d), especially when compared with the climatology for the same periods (Figs. $7 \mathrm{~g}$ and $7 \mathrm{~h}$ ). While a portion of the migration in the location of convection is certainly due to the seasonal shift in the ITCZ, the pattern of convection during JFMA 1998 exhibits a clear departure from climatology. Comparison of MERRA-analyzed OLR with CERESobserved OLR over these periods reveals MERRA to be very consistent with the observed features, though plots of the MERRA-CERES OLR difference (Figs. 7e and 7f) reveals MERRA to be biased high over the central Pacific Ocean and a bit low over the western Pacific warm pool, South Pacific convergence zone, and Central America. The mean MERRA-CERES bias is $3.52 \mathrm{~W} \mathrm{~m}^{-2}$ for January-April and $1.86 \mathrm{~W} \mathrm{~m}^{-2}$ for MayAugust, while the root-mean-squared error is 13.22 and $14.82 \mathrm{~m}^{-2}$ for each period, respectively. The results are in agreement with the analysis presented in Bosilovich et al. (2011), who note that the MERRA long-term mean OLR appears to be biased slightly high compared with values reported in Trenberth et al. (2009).

\section{b. Comparison between MERRA and cloud objects}

Deep convective cloud object data contains PDFs of OLR, outgoing (reflected) shortwave radiation (OSR), optical depth, cloud-top temperature, and liquid and ice water path for clouds determined to be associated with large-scale organized deep convection. Having verified that MERRA reproduced the gross features of the observed pattern of convection and large-scale circulation in 1998, we now conduct a more detailed comparison with the CERES cloud object data. Our aim is to assess 

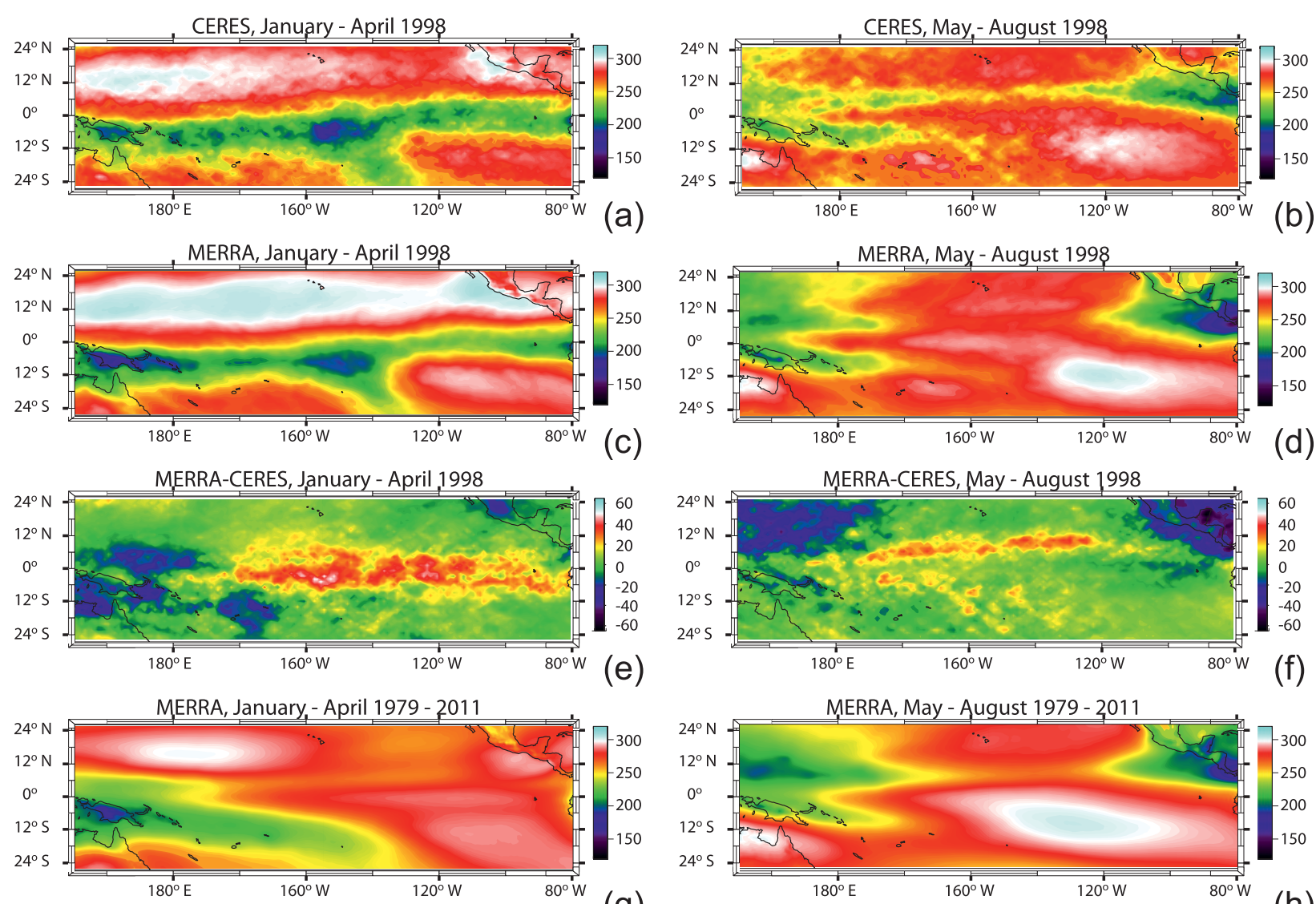

(g)

FIG. 7. (a),(b) CERES OLR compared with (c),(d) MERRA OLR averaged over (a),(c),(e),(g) January-April and (b),(d),(f),(h) MayAugust. Plots of the CERES-MERRA bias are depicted in (e) and (f), while plots of the mean JFMA and MJJA OLR averaged over the 1979-2011 period are shown in $(\mathrm{g})$ and $(\mathrm{h})$. In each plot, OLR has units of $\mathrm{W} \mathrm{m}^{-2}$.

MERRA-simulated cloud physical [total water path (TWP), cloud-top temperature] and radiative (optical depth, OLR, OSR) properties during the 1998 El NiñoLa Niña transition.

Comparison of MERRA and cloud object data for all object locations, sizes, and times is presented in Fig. 8. The shapes of the PDFs and their ranges of values are similar between reanalysis and observations, indicating that the MERRA is producing deep convection where it is observed in the CERES data. Compared with the observations, the total water path (Fig. 8a) in the MERRA is biased high with a mode at approximately $500 \mathrm{~g} \mathrm{~m}^{-2}$, while the observations exhibit a mode at approximately $300 \mathrm{~g} \mathrm{~m}^{-2}$. There are also discrepancies between the reanalysis and observations in the tail of the distribution; while the many of the observed objects exhibit TWP values in excess of $1400 \mathrm{~g} \mathrm{~m}^{-2}$, TWP in MERRA is uniformly lower than $1400 \mathrm{~g} \mathrm{~m}^{-2}$. As in the observations, most of the MERRA subcolumns have relatively low $(<40)$ optical depth (Fig. 8b), but there is a distinct lack of subcolumns with optical depth larger than 40 . Note that the spike at the optical depth of 128 in the observations is because 128 is defined to be the maximum optical depth in the VIRS cloud property retrieval algorithm (Minnis et al. 2011a,b). Cloud-top temperature is biased low, as is the outgoing longwave radiation, though it is notable that, while MERRA has no cloud-top temperature values lower than the lowest observed value, the reanalysis appears to be missing the lowest values of OLR. This could be because there is no way to represent overshooting convection (deep convection with tops that extend higher than the level of neutral buoyancy) in the Goddard Earth Observing System model, version 5 (GEOS-5). The mode in the PDF of outgoing shortwave radiation closely matches the observations, but MERRA contains too many columns with midrange OSR (400-700 $\left.\mathrm{W} \mathrm{m}^{-2}\right)$ and too few with OSR larger than $800 \mathrm{~W} \mathrm{~m}^{-2}$. Again, this may indicate difficulty representing deep convective cores in the subgrid comparison. We examine this in more detail later.

Cloud that is too high and thin (both physically and optically) with too few reflective and optically thick 

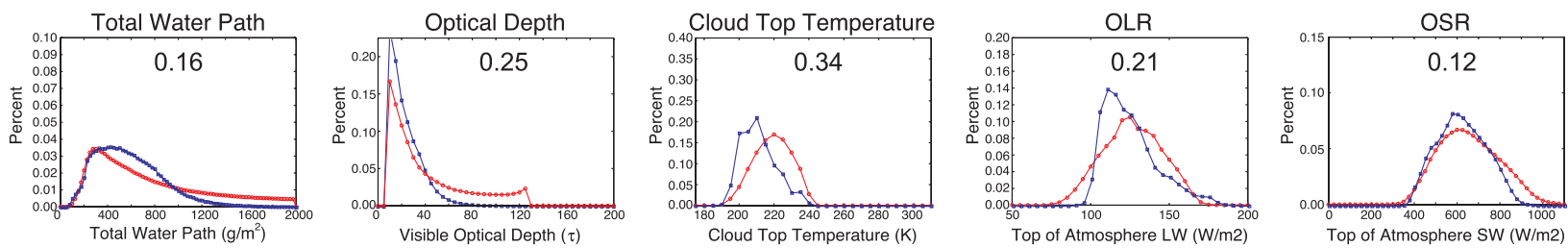

FIG. 8. Comparison of statistics from MERRA-simulated objects (blue) with CERES observations (red) for (a) TWP, (b) optical depth, (c) cloud-top temperature, (d) OLR flux, and (e) OSR flux. The numerical values in each plot correspond to $0.5 \times$ the integrated absolute difference between histograms (see text for details).

convective cores raises the question of whether the statistics might simply be reflecting an inability of the reanalysis to represent a particular size of object. For example, it is conceivable that the largest and most well-organized convective clusters might be better characterized by the reanalysis, while smaller clusters may have been missed (e.g., Xu 2009). We examine whether this may be the case by subsetting the dataset according to object size (Fig. 9). Consistency between analyzed and observed PDFs is quantified by computing the integrated absolute difference (IAD) between histograms, which can be expressed for $N$ histogram bins as

$$
\mathrm{IAD}=\sum_{i=1}^{N}\left|f\left(x_{\mathrm{obs}}\right)_{i}-f\left(x_{\text {MERRA }}\right)_{i}\right|
$$

where $f(x)_{i}$ is the histogram value in the $i$ th bin. The IAD is similar to an L1 norm, and as such is less sensitive to outliers than the root-mean-squared error (L2 norm). When multiplied by a factor of 0.5 , the IAD has the added benefit of an intuitive interpretation; IAD of 1.0 indicates a $100 \%$ difference between histograms (no shared probability mass), IAD of 0.5 indicates a $50 \%$ difference, etc. Note that the IAD could also be used as a measure of distance in a bootstrap test for significance in the assessment of differences between histograms (e.g., Xu 2006). The numerical values plotted inside each of Figs. 7-10 are the IAD for each MERRA-cloud object comparison.

As in Xu's (2009) comparison with ECMWF data, there is systematic improvement in the fit between MERRA and observations with increasing object size

\section{Object Size 300+ km}
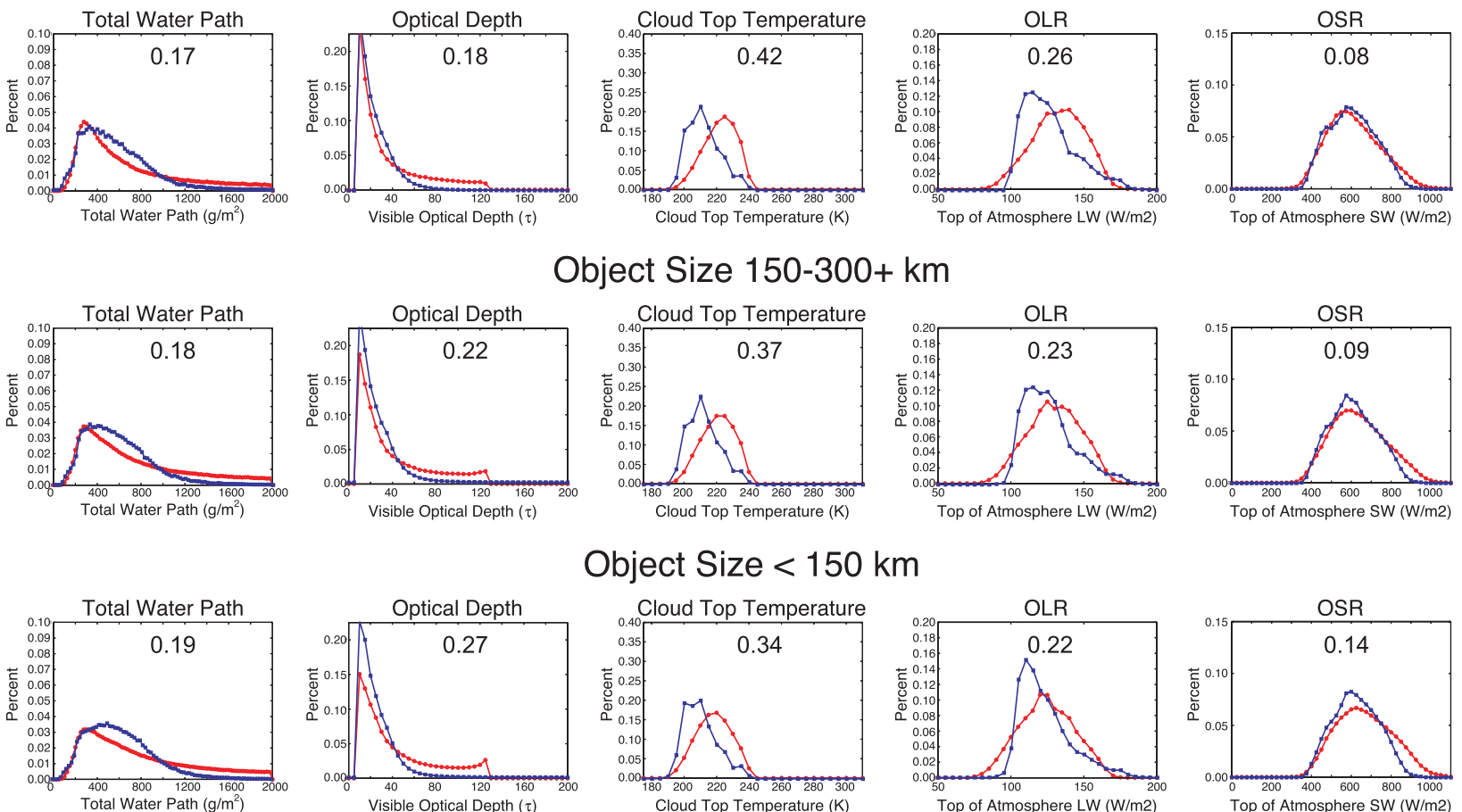

Object Size $<150 \mathrm{~km}$
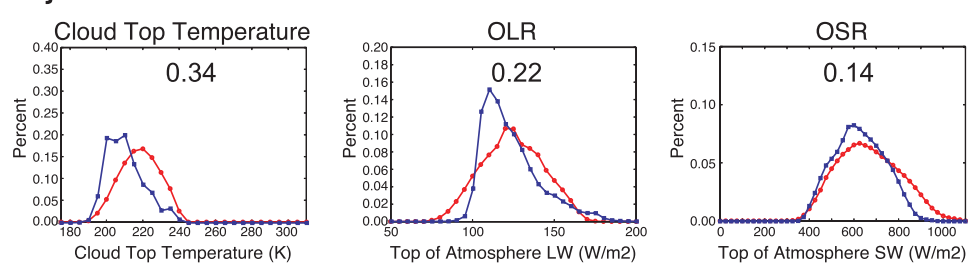

FIG. 9. As in Fig. 8, but for objects with diameters between (first row) 100 and 150, (second row) 150-300, and (third row) >300 km. 

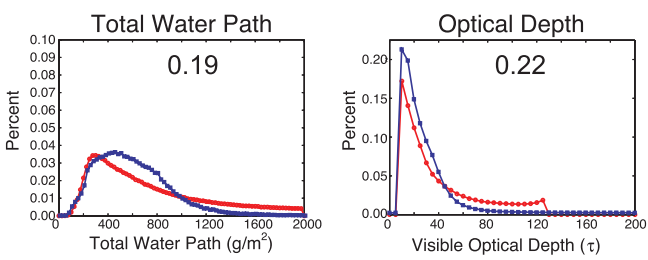

January - April 1998
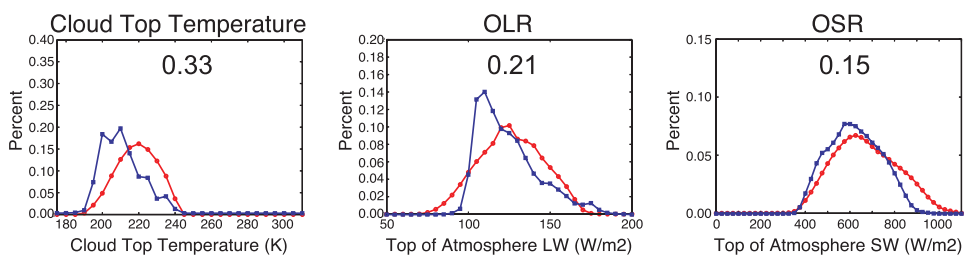

\section{May - August 1998}
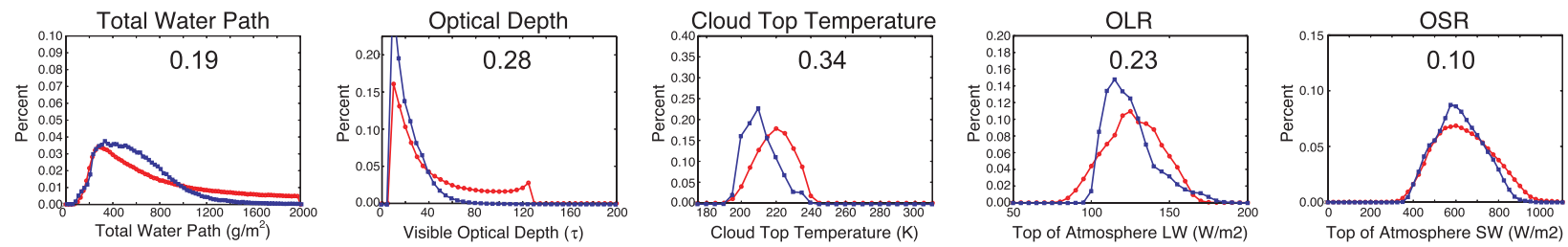

FIG. 10. Each row as in Fig. 8, but for objects observed during the (top) January-April 1998 time frame and (bottom) May-August 1998 time frame.

for the cloud-top temperature and OLR. In contrast, the fit degrades with object size for total water path, optical depth, and OSR. This appears to be because, while the frequency of occurrence of footprints with relatively low $(<40)$ optical depth decreases systematically with increasing object size [consistent with results presented in Xu et al. (2007) and Eitzen et al. (2009)], the frequency remains approximately the same in MERRA. Stated another way, cloud object PDFs are increasingly skewed toward large values of optical depth, TWP, and OSR with increasing size, while the shape of the MERRA PDFs remains relatively unchanged. Similarly, while the mode of the object histograms shifts toward higher OLR and cloud-top temperature with increasing object size, the mode of the MERRA PDFs remains fixed at approximately the same value. Improved consistency between MERRA and cloud object PDFs with increasing size is thus primarily due to a decrease in the number of MERRA subcolumns with relatively large values of OLR and cloud-top temperature. In the observations, smaller objects are characterized by relatively larger numbers of footprints with small physical and optical thickness, generally higher OLR and cloud-top temperature, and smaller values of OSR. A similar, but weaker, trend with object size can be seen in the MERRA histograms-larger objects have physically and optically thicker cloud, as well as lower cloud-top temperature and OLR and larger OSR. Interestingly, Eitzen et al. (2009) note that, when deep convective cloud objects are extended to include clouds with an optical depth less than 10, this trend is reversed; large objects have relatively greater amounts of thin cirrus relative to convective cores.

We now examine whether there are systematic differences between El Niño and La Niña conditions in the reanalysis. Comparison of PDFs for January-April 1998 (El Niño) and May-August 1998 (La Niña) (Fig. 10) reveal mixed results. There is no significant difference for PDFs of TWP, cloud-top temperature, or OLR. MERRA does produce a better fit to the observed optical depth under El Niño conditions and an improved match with cloud object OSR after the transition to La Niña. Overall, there is no systematic change in the performance of MERRA with the transition from El Niño to La Niña.

The picture that emerges from the MERRA-cloud object comparison is one in which MERRA produces too much stratiform anvil cloud and too little convective cloud. Stratiform cloud appears to be in general too physically thick, as represented in the overabundance of cloud with a TWP between 300 and $900 \mathrm{~g} \mathrm{~m}^{-2}$, while the cloud in deep convective cores appears to be too physically thin, reflected in the lack of large values of TWP, optical depth, and OSR, as well as the lack of the lowest values of OLR. However, we have made assumptions in the transformation from model to observation space, and before drawing conclusions it is useful to assess the robustness of the comparison.

\section{Sensitivity to assumptions used in the comparison}

Satellites observe the planet at generally higher spatial resolution than GCM model grid boxes. As a consequence, effective comparison between model and observations in the space of the observations necessitates disaggregation of each GCM grid box into subcolumns with appropriate subgrid variability. In the case of clouds, most of the attention in the literature has focused on 
evaluating sensitivity to cloud overlap assumptions, and it has led to the development of sophisticated algorithms that account for the vertical correlations between layers (Raisanen et al. 2004), and in some cases the multivariate relationship between cloud overlap and the environment (Larson 2007; Norris et al. 2008). The assumption of how subgrid condensate mass is parsed among subcolumns has received relatively less attention, but as we will show, it can have a significant impact on the results of the comparison.

In the above discussion, we made the standard assumption that cloud mass is distributed evenly among subgrid columns. This is the assumption made in the COSP (Bodas-Salcedo et al. 2008) and in Xu's (2009) evaluation of the ECMWF operational and reanalysis fields. Since the comparison at hand deals solely with deep convective clouds, we choose to modify the assumed distribution of subgrid condensate consistent with observations and high-resolution modeling studies of deep convection. As such, instead of assuming condensate is evenly distributed among subcolumns, we first sort the columns in order of decreasing depth of cloud (as measured by the number of vertical levels output from the overlap scheme flagged as containing cloud) and then exponentially weight the cloud mass between columns such that the deepest clouds receive the largest amount of condensate mass while the shallowest clouds receive the least. This is consistent with the notion that the cloud mass is likely to be concentrated in and near the convective core, with relatively less being found in the stratiform rainfall and cirrus anvil region. The exponential weight for subcolumn $i$ is computed according to the following decay equation:

$$
w\left(x_{i}\right)=\exp \left(-\frac{3}{N} x_{i}\right),
$$

where $N$ is the number of cloudy subcolumns and each weight was divided by the total so that the weights sum to one. We have chosen the decay rate to correspond to offline tests of the decrease in cloud condensate away from convective cores in long-term, large domain cloudresolving model simulations of organized tropical deep convection (Posselt et al. 2012), and the dependence on $N$ makes the rate of decay inversely proportional to the number of cloudy subcolumns. As with the evenly distributed condensate, the grid mean cloud mass mixing ratio is concentrated in only those subcolumns already determined to contain cloud.

To provide a baseline test of the sensitivity to the distribution of condensate across subcolumns, we perform two additional comparisons. In the first, we assume clouds are maximally overlapped instead of maximum-random overlap. In the second, we modify the assumed IWP-ice effective diameter relationship using Heymsfield et al's (2003) tropics-only functional fit. In both of these tests, condensate mass is evenly distributed among subcolumns, and the results are presented in Fig. 11. Exponential weighting of cloud mass across the MERRA subcolumns results in a reduction in the number of nondetected objects from 55 to 37 (1.25\% of the total number of cloud objects). It appears that this is largely due to an increase in the total number of deep convective subcolumns, as the number of objects for which MERRA produces an underestimate in the number of deep convective columns decreases, while the number of overestimated subcolumns increases (Table 2).

Application of an exponential distribution of cloud mass across the MERRA subcolumns has the effect of concentrating mass in convective columns and removing it from stratiform columns. The result is a shift in the mode of TWP toward smaller values and a significant increase in large values as compared with the even weighting (Fig. 8). There is a corresponding reduction in the number of subcolumns with small optical depth and an increase in those with large optical depth. The peak in the OSR at moderate values is reduced without sacrificing the fit at lower values, and the number of subcolumns with large OSR increases. The general result of a change to exponential condensate weighting is a nearperfect match between MERRA and cloud objects for $T W P$, optical depth, and OSR. In contrast, the fit between MERRA and cloud object OLR and cloud-top temperature worsens slightly with change to exponential weighting. Comparison with Fig. 8 reveals this is due to a decrease (increase) in the number of columns with relatively large (small) OLR and cloud-top temperature. This is consistent with an increase in the number of columns with relatively large optical depth and total water path. It should be noted that the improvements in the PDFs of TWP, optical depth, and OSR are greater than the degradation in the fit for OLR and cloud-top temperature. Because changes in the condensate weighting affect primarily the horizontal distribution of cloud and leave the location of cloud-top unchanged, there is less sensitivity to condensate weighting in the OLR and cloud-top temperature.

A comparison of the results produced using maximum overlap and those using maximum-random overlap reveals relatively little sensitivity to the overlap assumption, though it should be noted that maximum and maximumrandom overlap assumptions are very similar conceptually. Compared with evenly weighted condensate and maximum-random overlap, use of maximum overlap produces a slightly better fit to observations for all but the total water path. Use of a different IWP $-D_{i}$ 


\section{Exponentially Distributed Condensate}
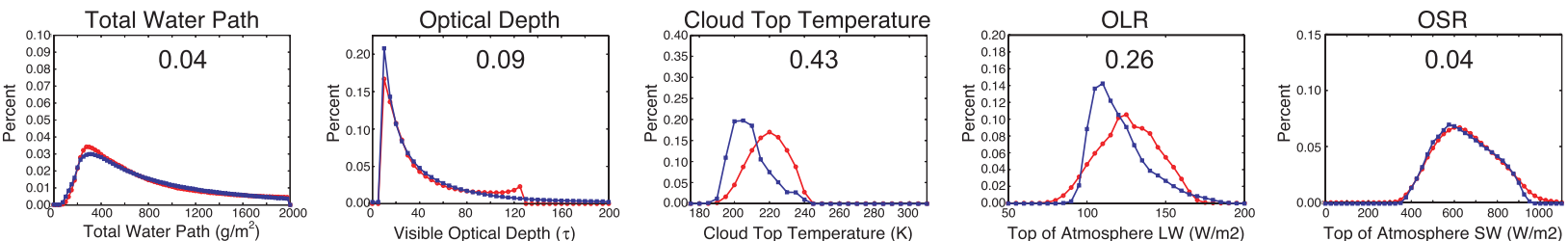

\section{Maximum Overlap}
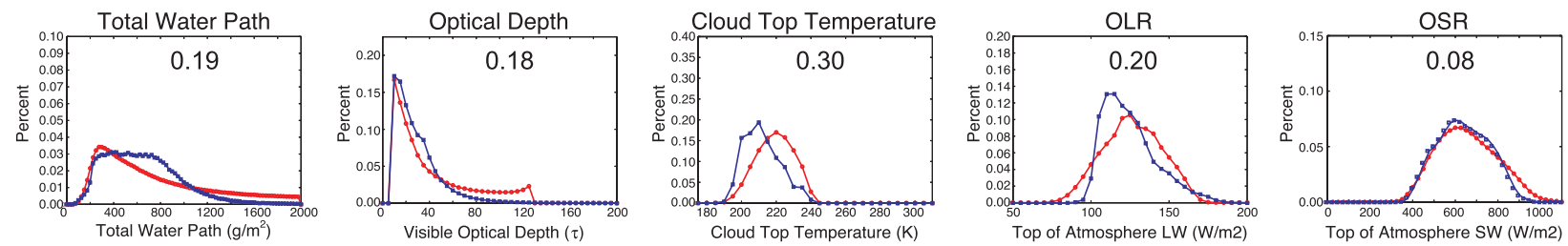

\section{Tropical IWP - Dei Relationship}
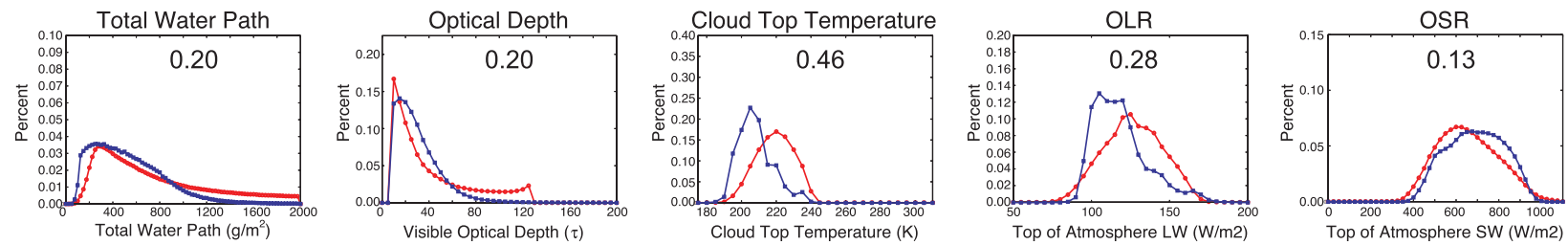

FIG. 11. As in Fig. 8, but in this case MERRA-simulated objects were produced using (top row) an assumed exponential distribution of condensate among subcolumns sorted according to cloud depth, (center row) maximum overlap instead of maximum-random overlap, and (bottom row) a modified relationship between IWP and ice effective diameter (see text for details).

relationship has a relatively large effect on the comparison for nearly all variables. There is a shift in the TWP toward smaller values, as well as a shift in OSR toward higher values. OLR is biased lower as is the cloud-top temperature. This is likely due to the tropical deep convective clouds Heymsfield et al. (2003) used to generate this functional fit all had optical depths greater than about 20 . The tropical IWP $-D_{i}$ relationship was found to produce a much smaller effective diameter for small values of IWP compared with the relationship obtained from the combined dataset.

\section{Summary and conclusions}

In this paper, deep convective clouds in the MERRA reanalysis were compared with TRMM-observed deep convective cloud objects for the 1998 El Niño-La Niña transition. We assessed MERRA's representation of cloud content, optical thickness, and radiative properties, and conducted sensitivity tests to determine the robustness of the comparison. We find that MERRA accurately reproduces the time mean observed TOA OLR and realistically represents the observed shift in the ascending branch of the Walker circulation from the central to the western Pacific. MERRA was found to contain deep convective cloud in $98.1 \%$ of the observed cloud object cases, and it produced PDFs of cloud and radiative variables with forms that are consistent with those in the observations.

While many of the general cloud properties are congruent between the reanalysis and CERES cloud objects, deep convective clouds in MERRA appear to be too optically and physically thin, with cloud top biased high and cold. The consistency between MERRA and observations improves with increasing convective system size for OLR and cloud-top temperature, with the best fit found for the largest deep convective systems. The finding that OLR is biased low in MERRA compared with the CERES cloud objects runs counter to the long-term mean behavior of MERRA (Bosilovich et al. 2011), and to our own comparisons of MERRA with

TABLE 2. As in Table 1, but for statistics generated using an exponential distribution of condensate mass. See text for details.

\begin{tabular}{|c|c|c|c|c|}
\hline \multirow[b]{2}{*}{ Threshold } & \multicolumn{2}{|c|}{ Underestimate (\%) } & \multicolumn{2}{|c|}{ Overestimate $(\%)$} \\
\hline & $<10$ & $<20$ & $>150$ & $>200$ \\
\hline Small & 2.74 & 3.49 & 29.75 & 28.09 \\
\hline Medium & 1.93 & 3.08 & 28.26 & 25.52 \\
\hline Large & 0.85 & 1.29 & 13.37 & 10.15 \\
\hline
\end{tabular}


CERES 4-month means. This is likely because our comparison focuses only on the deepest and most optically thick clouds, while the long-term means include all clouds. The cold bias we observe is likely to be masked by warm biases in regions of clear air or optically thin cloud when temporal averages are computed. This ability to extract information associated with specific cloud types is one of the key strengths of an object-oriented analysis.

In contrast to OLR and cloud-top temperature, MERRA-analyzed TWP, optical depth, and OSR were found to exhibit good agreement with CERES objects. Interestingly, MERRA has been shown to be dry compared with other reanalysis datasets (A. Molod 2011, personal communication). As with the OLR and cloudtop temperature, these comparisons are performed for time means that include the full spectrum of clouds as well as clear air. Restricting the comparison to deep convective clouds allows us to evaluate a specific set of processes in the analysis. Our results suggest that the dry bias noted in comparisons with other reanalysis datasets is not due to a lack of condensate in deep convective clouds.

The results of our comparison are found to be sensitive to assumptions made in the disaggregation of gridscale cloud to satellite pixel scales. When cloud mass is weighted exponentially (rather than evenly) across subcolumns, the result is a reduction in the number of deep convective clouds missed in MERRA to $1.3 \%$, and a near-perfect fit between MERRA and objects for TWP, optical depth, and OSR. In fact, we find that changes in the weighting scheme have a larger effect on the outcome of the comparison than do changes to the cloud overlap scheme or the assumed IWP-Di relationship. This has implications for the robustness of comparisons between GCM and reanalysis data and satellite observations, especially for cases that require specification of an assumed subgrid distribution of cloud mass (as is required in satellite simulator packages). Our results motivate a careful exploration of not only the differences between models and observations but also of the uncertainties inherent in the comparison itself. That said, it should be noted that model-observation differences in cloud-top temperature and OLR are robust to changes in the assumptions used in the subgrid cloud scheme. In addition, it is not clear whether model-data comparisons of other cloud types (e.g., stratocumulus) would exhibit a similar degree of sensitivity.

Finally, it is possible that the robust cold bias seen in comparisons of cloud-top temperature and OLR is due to an overabundance of upper-tropospheric condensate detrained from the Relaxed Arakawa-Schubert (RAS; Moorthi and Suarez 1992) convective scheme. Work is currently being done on a modified version of the RAS scheme that allows for enhanced detrainment at lower levels (C. Anderson 2011, personal communication). Future work also includes use of deep convective objects to evaluate GCM-simulated deep convection. As opposed to MERRA, GCMs are not constrained to reproduce clouds at their observed time and location. We describe a method of comparison that can be used in this case, and report the results of such a comparison in a future study.

Acknowledgments. This research was supported by the NASA Modeling, Analysis, and Prediction program under Grants NNX08AF77G and NNX09AJ43G. CERES cloud object data were obtained from the NASA Langley Research Center (at http://cloud-object.larc. nasa.gov). The MERRA data used in this research were acquired as part of the activities of NASA's Science Mission Directorate, and are archived and distributed by the Goddard Earth Sciences (GES) Data and Information Services Center (DISC). Andrea Molod provided valuable feedback on the comparison results and assistance with the GEOS-5 model and physical parameterizations. Discussions with Kuan-Man $\mathrm{Xu}$ and the comments of three anonymous reviewers greatly improved the analysis and interpretation of the cloud object comparison results.

\section{REFERENCES}

Bell, G. D., M. S. Halpert, C. F. Ropelewski, V. E. Kousky, A. V. Douglas, R. C. Schnell, and M. E. Gelman, 1999: Climate assessment for 1998. Bull. Amer. Meteor. Soc., 80, S1-S48.

Bodas-Salcedo, A., M. J. Webb, M. E. Brooks, M. A. Ringer, K. D. Williams, S. F. Milton, and D. R. Wilson, 2008: Evaluating cloud systems in the Met Office global forecast model using simulated CloudSat radar reflectivities. J. Geophys. Res., 113, D00A13, doi:10.1029/2007JD009620.

Bosilovich, M. G., F. R. Robertson, and J. Chen, 2011: Global energy and water budgets in MERRA. J. Climate, 24, 5721-5739.

Eitzen, Z. A., K.-M. Xu, and T. Wong, 2009: Cloud and radiative characteristics of tropical deep convective systems in extended cloud objects from CERES observations. J. Climate, 22, 59836000.

Fu, Q., and K.-N. Liou, 1992: On the correlated $k$-distribution method for radiative transfer in nonhomogeneous atmospheres. J. Atmos. Sci., 49, 2139-2156.

Heymsfield, A. J., S. Matrosov, and B. Baum, 2003: Ice water pathoptical depth relationships for cirrus and deep stratiform ice cloud layers. J. Appl. Meteor., 42, 1369-1390.

Kalnay, E., and Coauthors, 1996: The NCEP/NCAR 40-Year Reanalysis Project. Bull. Amer. Meteor. Soc., 77, 437-471.

Kummerow, C., W. Barnes, T. Kozu, J. Shiue, and J. Simpson, 1998: The Tropical Rainfall Measuring Mission (TRMM) sensor package. J. Atmos. Oceanic Technol., 15, 809-817.

Larson, V. E., 2007: From cloud overlap to PDF overlap. Quart. J. Roy. Meteor. Soc., 133, 1877-1891.

Manton, M. J., and W. R. Cotton, 1977: Formulation of approximate equations for modeling moist deep convection on the 
mesoscale. Colorado State University Atmospheric Science Paper 266, 62 pp.

Minnis, P., and Coauthors, 1997: Cloud optical property retrieval (subsystem 4.3). Clouds and the Earth's Radiant Energy System (CERES) algorithm theoretical basis document, $60 \mathrm{pp}$. [Available online at http://ceres.larc.nasa.gov/atbd.php.]

— , and Coauthors, 2011a: CERES edition-2 cloud property retrievals using TRMM VIRS and Terra and Aqua MODIS data-Part I: Algorithms. IEEE Trans. Geosci. Remote Sens., 49, 4374-4400.

— , and Coauthors, 2011b: CERES edition-2 cloud property retrievals using TRMM VIRS and Terra and Aqua MODIS data-Part II: Examples of average results and comparisons with other data. IEEE Trans. Geosci. Remote Sens., 49, 44014430.

Moorthi, S., and M. J. Suarez, 1992: Relaxed Arakawa-Schubert, a parameterization of moist convection for general circulation models. Mon. Wea. Rev., 120, 978-1002.

Norris, P. M., L. Oreopoulos, A. Y. Hou, W.-K. Tao, and X. Zeng, 2008: Representation of 3D heterogeneous cloud fields using copulas: Theory for water clouds. Quart. J. Roy. Meteor. Soc., 134, 1843-1864.

Onogi, K., and Coauthors, 2005: JRA-25: Japanese 25-Year ReAnalysis Project-Progress and status. Quart. J. Roy. Meteor. Soc., 131, 3259-3268.

Posselt, D. J., S. C. van den Heever, G. L. Stephens, and M. R. Igel, 2012: Changes in the interaction between tropical convection, radiation, and the large-scale circulation in a warming environment. J. Climate, 25, 557-571.

Raisanen, P., H. W. Barker, M. F. Khairoutdinov, J. Li, and D. A. Randall, 2004: Stochastic generation of subgrid-scale cloudy columns for large-scale models. Quart. J. Roy. Meteor. Soc. 130, 2047-2067.

Rienecker, M. M., and Coauthors, 2008: The GEOS-5 Data Assimilation System-Documentation of versions 5.0.1 and 5.1.0, and 5.2.0. NASA Technical Report Series on Global Modeling and Data Assimilation, Vol. 27, NASA Tech Memo. NASA/ TM-2008-104606, 101 pp.
- , and Coauthors, 2011: MERRA: NASA's Modern-Era Retrospective Analysis for Research and Applications. J. Climate, 24, 3624-3648.

Robertson, F. R., M. G. Bosilovich, J. Chen, and T. L. Miller, 2011: The effect of satellite observing system changes on MERRA water and energy fluxes. J. Climate, 24, 5197-5217.

Stephens, G. L., 2005: Cloud feedbacks in the climate system: A critical review. J. Climate, 18, 237-273.

Trenberth, K. E., J. T. Fasullo, and J. Kiehl, 2009: Earth's global energy budget. Bull. Amer. Meteor. Soc., 90, 311-323.

Uppala, S. M., and Coauthors, 2005: The ERA-40 Re-Analysis. Quart. J. Roy. Meteor. Soc., 131, 2961-3012.

Wielicki, B. A., B. R. Barkstrom, E. F. Harrison, R. B. Lee III, G. L. Smith, and J. E. Cooper, 1996: Clouds and the Earth's Radiant Energy System (CERES): An Earth Observing System experiment. Bull. Amer. Meteor. Soc., 77, 853-868.

$\mathrm{Xu}, \mathrm{K} .-\mathrm{M} ., 2006$ : Using the bootstrap method for a statistical significance test of differences between summary histograms. Mon. Wea. Rev., 134, 1442-1453.

, 2009: Evaluation of cloud physical properties of ECMWF analysis and re-analysis (ERA) against CERES tropical deep convective cloud object observations. Mon. Wea. Rev., 137, 207-223.

, T. Wong, B. A. Wielicki, L. Parker, and Z. A. Eitzen, 2005: Statistical analyses of satellite cloud object data from CERES. Part I: Methodology and preliminary results of the $1998 \mathrm{El}$ Niño/2000 La Niña. J. Climate, 18, 2497-2514.

, B. Lin, Z. A. Eitzen, and M. Branson, 2007: Statistical analyses of satellite cloud object data from CERES. Part II: Tropical convective cloud objects during 1998 El Niño and evidence for supporting the fixed anvil temperature hypothesis. J. Climate, 20, 819-842.

,,-- - and,- 2008 : Statistical analyses of satellite cloud object data from CERES. Part IV: Boundary layer cloud objects during 1998 El Niño. J. Climate, 21, 1500-1522.

Yeh, S.-W., J.-S. Kug, B. Dewitte, M.-H. Kwon, B. P. Kirtman, and F.-F. Jin, 2009: El Niño in a changing climate. Nature, 461, 511-515. 